\title{
LA LOMIDINE DANS LE TRAITEMENT DE LA THEILERIOSE BOVINE
}

\author{
par J. GRIMPRET
}

En Juin 1947, sur les indications du Laboratoire de Recherches du Service de l'Élevage à Cạsablanca, un nouveau produit a été expérimenté, dans le traitement de la Theileriose.

Il s'agit de la Lomidine, dérivé de la Guanidine, une poudre granuleuse blanche, à odeur butyrique, très hygroscopique et assez soluble dans l'eau.

Ce médicament s'emploie en solution à $5 \%$ qu'il est indispensable de préparer extemporanément avec de l'eau tiède. La nécessité de chauffage est un petit inconvénient à l'utilisation pratique du produit.

La dose est de 3 milligrammes par kilogramme de poids yif, ce qui correspond à 20 ou $30 \mathrm{cc}$. de solution pour les bovidés marocains de poids moyen. L'injection faite en intraveineuse est toujours très bien supportée par le malade; il n'y a jamais de réaction. Là voie intramusculaire peut être employée aussi avec profit.

Voici les résultats obtenus:

$1^{\circ}$ Tràitement pár la " Lomidine " seule - L Les bovidés atteints de Theileriose présentent d'emblée une très forte fièvre. La Lomidine, dans tous les cas, produit rapidement une baisse sensible de la température. Sur tous les animaux hospitalisés et dont la température pouvait être contrôlée toutes les demi-heures, il a été constaté que la fièvre baissait toujours nettement, cinq heures après l'injection intraveineuse de Lomidine.

Lorsque le traitement est précoce, le seul fait d'agir aussi brutalement sur la fièvre donne au malade la possibilité de mieux se défendre, et on peut alors obtenir la guérison clinique de la Theileriose bovine avec la Lomidine, après 1 ou 2 injections.

Les guérisons de cet ordre sont malheureusement peu fréquentes. Dans la majorité des cas, la baisse de température n'est pas suffisante pour que le malade puisse récupérer et lutter victorieusement contre les Theileria. Une nouvelle poussée thermique apparaît très vite.: La seconde injection de Lomidine produit le même effet que la première, soit une baisse passagère de la température qui remonte bientôt; et ainsi de suite, donnant une courbe en dents de scie très particulière.

Elev, et Méd. vét. des Pays tropicaux. - Oct.-Déc. 1948. 
Ces grandes oscillations de lempéralure conduisent dans tous lés cas à la mort du malade, avec aggravation des symptômes cliniques à chaque pointe fébrile.

Malgré la répétition des injections, trois bovidés atteints de Theileriose sont morts successivement.

L'inconstance des résultats obtenus, et la mortalité enregistrée sur. les dix premiers cas traités, m'ont incité à ne plus employer la Lomidine seule, mais à la combiner avec la Gonacrine, plus constante dans ses effets.

Toutefois, deux enseignements cliniques pouvaient déjà être valablement retenus, en faveur de la Lomidine:

a) La Lomidine est un antifébrile énergique et d'action rapide; plus actif, dans ce domaine, que l'Acetanilide, la Gonacrine ou le Zoothelone, couramment utilisés dans la thérapeutique des piroplasmoses.

L'ampleur des oscillations thermiques m'a fait supposer que l'action de la Lomidine était fugace, et qu'il était peut-être bon de maintenir le malade, plus longtemps sous l'action du médicament. Les injections intraveineuses ont alors été doublées par des injections intramuśculaires faites en même temps et aux mêmes doses. Les résultats n'ont pas été modifiés. La Lomidine est probablement très vite détruite ou éliminée par l'organisme.

b) La Lomidine esl un antitoxique. Les animaux atteints de Theileriose et traités par la Lomidine ne présentent jamais l'amaigrissement accusé, la fonte des tissus, qu'on observe régulièrement dans cette maladie. L'observation est valable, mème lorsque le malade succombe.

Dès 1943 (l), j'ai indiqué, à propos de l'emploi de la Gonacrine, qu'on' pouvait penser que ce médicament contrecarrait efficacement l'action de quelque toxine. Zotrner et Camou (2) estiment que la cause primordiale de la Theileriose bovine est cette toxine produite par les Theileria, ou par quelque désintégration globulaire.

Puisque la Lomidine fait baisser la fièvre et empêche la fonte des tissus, on peut logiquement supposer qu'elle agit contre les toxines en question.

Par contre, son aclion élanl passagère, on pout émettre l'hypothèse que la Lomidine n'a pas d'action sur les Theileria et que, par voie de conséquence, ce sont les Theileria qui émettent les toxines.

$2^{\circ}$ Traitement par la Lomidine et la Gonacrine - De nombreux bovidés atteints de Theileriose confirmée ont été soumis à l'action combinée de la-Lomidine et de la Gonacrine.

(1) J. Grimpret. - Traitement de la Theileriose Bovine. Maroc Médical 1943, page 61.

(2) G. Zotrnen ot R. Camou. - Theileriose, parasitismo et toxémie. Maroc Médical no 266; 1947, page 159. 
Les résultats ont été excellents et aucune mortalité n’a été enregistrée.

La Lomidine peut être injectée la première, en intraveineuse, et suivie quelques heures après d'une injection de Gonacrine, ou inversement.

Dans la pratique, le meilleur résultat est obtenu en injectant la Gonacrine dans la veine et, en mème temps, la même quantité de Lomidine ea intramusculaire.

La guérison clinique est souvent enregistrée en une seule intervention; il en faut rarement plus de deux. Dans tous les cas, les malades ont reçu également des toni-cardiaques et une méditation symptomatique.

Dans ces cas, la courbe thermique ne présente jamais des oscillations dont l'amplitude est observée quand la Lomidine est employée seule. La chute thermique est moins brutale, et la température revient régulièrement vers la normale.

L'utilisation combinée de la Lomidine et de la Gonacrine est donc tout à fait à conseiller. La Lomidine qui s'emploie en intramusculaire peut être déposée dans toute les fermes, et l'éleveur peut, à tout moment, faire utilement l'injection de Lomidine, en attendant le vétérinaire qui pratiquera l'intraveineuse de Gonacrine et formulera tout traitement symptomatique approprié. Il ne faut pas oublier l'importance de la rapidité d'intervention, en matière de Theileriose bovine.

Ces succès amènent les réflexions suivantes : dans le traitement de la Theileriose bovine, la Lomidine est un antifébrile et un antitoxique quii, employé seul, donne irrégulièrement des guérisons cliniques, parce que sans action sur les Theileria. Si on traite la même maladie par combinaison de Gonacrine et de Lomidine, les résultats favorables sont plus nombreux, plus constants et les animaux maigrissent peu. Ceci paraît montrer que Ia Gonacrine agit probablement à la fois sur la toxine et sur le parasite, mais davantage sur les Theileria que sur la toxine.

Du 20 Juin au 10 Septembre 1947, 57 bovidés atteints de Theileriose clinique ont été traités par la Lomidine, seule ou associée à la Gonacrine. Trois seulement ont succombé et tous les trois n'ont reçu que de la Lomidine.

Ges chiffres donnent une mortalité de moins $6 \%$, ce qui est remarquable, en matière de Theileriose bovine.

Un certain nombre de ces cas de Theileriose ont été contrôlés par le Laboratoire de Recherches du Service de l'Élevage, en ne tenant compte que des 25 cas pour lesquels le Laboratoire spécialisé a confirmé la présence de Theileria, et qui intéressent:

1 Schwytz

2 Montbéliards 


\author{
4. Tarentais \\ 1 Hollandais \\ 10 croisés Tarentais \\ 2 croisés. Montbéliards \\ 1.croisé Schwytz \\ 1 croisé Zébu \\ 3 Marocains
}

on a enregistré seulement 3 morts, 3 bovidés qui n'avaient reçu que de la Lomidine : 1 Schwytz, 1 Montbéliard, 1 Marocain. La mortalité est alors de $12 \%$.

Faute de Lomidine, l'expérimentation a été interrompue le 10 Scptembre.

Conclusions. - Il se confirme que la chimiothérapie est une arme sérieuse pour lutter contre la Theileriose bovine.

La Lomidine est un médicament nouveau qui a sa place dans l'arsenal thérapeutique du praticien.

Vis-à-vis de la Theileriose bovine, la Lomidine se montre un "antifébrile et un antitoxique très intéressant. Utilisée seule, c'est un moyen de traitement insuffisant et inconstant dans ses effets.

Par contre, il paraît compléter heureusement l'action de la Gonacrine qui reste, pour l'instant, le médicament le plus efficace contre la Theileriose bovine.

L'emploi combiné de la Lomidine et de la Gonacrine est l'acheminemeńt vers le traitement idéal de la Theileriose, si magistralement exposé par Zotrner et Camou, à savoir : la Lomidine est un antitoxique associé à la Gonacrine qui apparâtt surtout comme un antiparasitaire. 